\title{
Average-case consistency measurement and analysis of interval-valued reciprocal preference relations
}

\author{
Yucheng Dong ${ }^{\mathrm{a}}$, Cong-Cong $\mathrm{Li}^{\mathrm{a}}$, Francisco Chiclana ${ }^{\mathrm{b}}$, Enrique Herrera-Viedma ${ }^{\mathrm{c}, \mathrm{d}}$ \\ ${ }^{a}$ Business School, Sichuan University, Chengdu, China \\ ${ }^{b}$ Centre for Computational Intelligence, Faculty of Technology, De Montfort University, Leicester, UK \\ ${ }^{c}$ Department of Computer Science and Artificial Intelligence, University of Granada, Granada, Spain \\ ${ }^{d}$ Department of Electrical and Computer Engineering, Faculty of Engineering, King Abdulaziz University, Jeddah, \\ Saudi Arabia
}

\begin{abstract}
Measuring consistency of preferences is very important in decision-making. This paper addresses this key issue for interval-valued reciprocal preference relations. Existing studies implement one of two different measures: the "classical" consistency measure, and the "boundary" consistency measure. The classical consistency degree of an interval-valued reciprocal preference relation is determined by its associated reciprocal preference relation with highest consistency degree, while the boundary consistency degree is determined by its two associated boundary reciprocal preference relations. However, the consistency index of an interval-valued reciprocal preference relation should be determined by taking into account all its associated reciprocal preference relations. Motivated by this, a new consistency measure for interval-valued reciprocal preference relations, the average-case consistency measure, is suggested and introduced. The new average-case consistency measure of an interval-valued reciprocal preference relation is determined as the average consistency degree of all reciprocal preference relations associated to the interval-valued reciprocal preference relation. Furthermore, the analysis and comparison of the different consistency measure internal mechanisms is used to justify the validity of the average-case consistency measure. Finally, an average-case consistency improving method which aims to obtain a modified interval-valued reciprocal preference relation with a required average consistency degree is developed.
\end{abstract}

Keywords: Decision analysis, reciprocal preference relation, interval-valued preferences, consistency measurement, average-case consistency.

\section{Introduction}

Reciprocal preference relations are based on the pairwise comparison method, and are widely used preference representation structures in decision-making problems. Various types of reciprocal preference relations have been proposed, such as additive preference relations (also called fuzzy preference relations) $[2,11,13,14,24]$, and multiplicative preference relations [3, 21-23]. It is well known that

Email addresses: ycdong@scu.edu.cn (Yucheng Dong), congcongli@stu.scu.edu.cn (Cong-Cong Li), chiclana@dmu.ac.uk (Francisco Chiclana), viedma@decsai.ugr.es (Enrique Herrera-Viedma) 
quantifying consistency is a very important issue in decision-making with preference relations. The lack of consistency can lead to inconsistent conclusions. In the specialised literature, a number of consistency measurement methods of reciprocal preference relations have been proposed (see, among others, $[1,7,15,17,18,34,38])$.

However, due to the complexity and uncertainty involved in real-world decision problems, it is sometimes unrealistic to acquire exact judgments. Thus, reciprocal preference relations are extended to interval-valued reciprocal preference relations (see, among others, $[27,35]$ ). In this paper, we focus on the consistency of interval-valued reciprocal preference relations. Existing studies regarding the measurement of consistency of interval-valued reciprocal preference relations can be broadly classified as implementing one of two different measures that we refer to as: the "classical" consistency measure $[8,12,27,35]$, and the "boundary" consistency measure [19, 20], which are described in Section 2.2. However, based on the definitions of the classical and boundary consistency measures (see Eqs. (3) and (4)), we can find that:

(1) The classical consistency degree of an interval-valued reciprocal preference relation is determined by its associated reciprocal preference relation with highest consistency degree, while

(2) The boundary consistency degree is determined by its two associated boundary reciprocal preference relations.

It is natural that the consistency index of an interval-valued reciprocal preference relation should be determined by taking into account all its associated reciprocal preference relations. Motivated by this, in this paper a new average-case consistency analysis of interval-valued reciprocal preference relations is suggested, defined and analysed. Furthermore, this paper also proposes an average-case consistency improving method, based on the relationship among the average-case consistency measure, the classical consistency measure, and the worst consistency measure.

The rest of the paper is organised as follows. Section 2 introduces a basic description of the interval-valued reciprocal preference relation, the classical consistency measure and the boundary consistency measure. Section 3 presents the average-case consistency analysis of the interval-valued reciprocal preference relation (Section 3.1), as well as a numerical analysis (Section 3.2) and the different consistency measure internal mechanisms (Section 3.3). Section 4 is dedicated to the averagecase consistency improving method. Finally, concluding remarks are included in Section 5.

\section{Preliminaries}

This section provides the basic knowledge regarding interval-valued reciprocal preference relations, as well as the classical consistency measure and the boundary consistency measure for interval-valued reciprocal preference relations. 


\subsection{Interval-Valued Reciprocal Preference Relations}

The definitions of both the additive reciprocal preference relation and the interval-valued additive reciprocal preference relation are given below.

Definition 1 (Additive Reciprocal Preference Relation [13, 23]). A matrix $F=\left(f_{i j}\right)_{n \times n}$, with $f_{i j} \in[0,1]$ and $f_{i j}+f_{j i}=1 \forall i, j \in\{1,2, \ldots, n\}$, is called an additive reciprocal preference relation.

Definition 2 (Interval-Valued Additive Reciprocal Preference Relation [35]). A matrix $\tilde{V}=$ $\left(\tilde{v}_{i j}\right)_{n \times n}$, with $\tilde{v}_{i j}=\left[v_{i j}^{-}, v_{i j}^{+}\right] \subseteq[0,1]$ and $v_{i j}^{-}+v_{j i}^{+}=1 \forall i, j \in\{1,2, \ldots, n\}$, is called an interval-valued additive reciprocal preference relation.

There are two main types of reciprocal preference relations: additive reciprocal preference relations and multiplicative reciprocal preference relations. The transformation functions between these types of reciprocal preference relations have been presented in [3], so this paper focuses entirely on intervalvalued additive reciprocal preference relations, and the proposed results can be similarly applied to interval-valued multiplicative reciprocal preference relations via the corresponding transformation function. In this paper, the additive reciprocal preference relation and the interval-valued additive reciprocal preference relation will be denoted simply as RPR and IVRPR, respectively.

Clearly the concept of IVRPR extends the concept of RPR, and when $v_{i j}^{-}=v_{i j}^{+} \forall i, j$ then an IVRPR becomes an RPR. However, when there exists at least a pair of values $(i, j)$ such that $v_{i j}^{-}<v_{i j}^{+}$ then an IVPR can be seen as a collection of (associated) RPRs as the following definition implies:

Definition 3 (RPRs associated to an IVRPR [8]). Let $\tilde{V}=\left(\tilde{v}_{i j}\right)_{n \times n}$, with $\tilde{v}_{i j}=\left[v_{i j}^{-}, v_{i j}^{+}\right] \subseteq$ $[0,1]$, be an IVRPR. An RPR $F=\left(f_{i j}\right)_{n \times n}$ that verifies

$$
v_{i j}^{-} \leq f_{i j} \leq v_{i j}^{+} \forall i, j \in\{1,2, \ldots, n\}
$$

is called an RPR associated to $\tilde{V}$. The set of all RPRs associated to an IVRPR $\tilde{V}$ is denoted by $N_{\tilde{V}}$.

Notice that given an IVRPR $\tilde{V}=\left(\tilde{v}_{i j}\right)_{n \times n}$, with $\tilde{v}_{i j}=\left[v_{i j}^{-}, v_{i j}^{+}\right] \subseteq[0,1], N_{\tilde{V}}$ contains the following two associated RPRs $B=\left(b_{i j}\right)_{n \times n}$ and $C=\left(c_{i j}\right)_{n \times n}$ :

$$
b_{i j}=\left\{\begin{array}{ll}
v_{i j}^{+} & i<j \\
0.5 & i=j, \\
v_{i j}^{-} & i>j
\end{array} \quad c_{i j}=\left\{\begin{array}{cc}
v_{i j}^{-} & i<j \\
0.5 & i=j . \\
v_{i j}^{+} & i>j
\end{array}\right.\right.
$$

In this paper the RPRs $B$ and $C$ are called the boundary RPRs associated to the IVRPR $\tilde{V}$. 


\subsection{Consistency Measures of IVRPRs}

In the following, we provide the definition of the consistency index of an RPR. The classical and boundary consistency measures of an IVRPR that, based on the consistency index of RPRs, have been proposed in the literature are also provided.

Consistency index of RPRs [15]. Based on the additive transitivity property [24], Herrera-Viedma et al. [15] proposed the following consistency index (CI) of an RPR F:

$$
C I(F)=1-\frac{4}{n \cdot(n-1) \cdot(n-2)} \sum_{i=1}^{n} \sum_{j=i+1}^{n} \sum_{k=j+1}^{n}\left|f_{i j}+f_{j k}-f_{i k}-0.5\right|
$$

The larger the value of $C I(F)$ the more consistent $F$ is. Generally, in practice decision makers may establish a consistency threshold $\overline{C I}$ for RPRs so that a given RPR $F$ that verifies $C I(F) \geq \overline{C I}$ is considered of acceptable consistency; otherwise, $F$ is considered of unacceptable consistency.

Meanwhile, to our knowledge, two kinds of consistency measures for IVRPRs have been presented based on the concept of consistency index of an RPR given in Eq.(2):

Classical consistency measure of IVRPRs $[\mathbf{1 2}, \mathbf{3 5}]$. Let $\tilde{V}$ be an IVRPR. If there exists an RPR associated to $\tilde{V}, F \in N_{\tilde{V}}$, such that $C I(F)=1$ then $\tilde{V}$ is considered to be consistent. In this paper, the classical consistency index (CCI) of an IVRPR $\tilde{V}$ is formally expressed as follows:

$$
C C I(\tilde{V})=\max _{F \in N_{\tilde{V}}} C I(F)
$$

Therefore, when $C C I(\tilde{V})=1, \tilde{V}$ is consistent; otherwise, $\tilde{V}$ is not consistent.

Boundary consistency measure of IVRPRs $[\mathbf{1 9}, \mathbf{2 0}]$. Let $\tilde{V}$ be an IVRPR. If its associated boundary RPRs given in Eq.(1), $B$ and $C$, are both of acceptable consistency, then $\tilde{V}$ is of acceptable consistency. In other words, if $C I(B) \geq \overline{C I}$ and $C I(C) \geq \overline{C I}$, then $\tilde{V}$ is of acceptable consistency; otherwise, $\tilde{V}$ is of unacceptably consistency. In this paper, the boundary consistency index (BCI) of an IVRPR $\tilde{V}$ is formally expressed as follows:

$$
B C I(\tilde{V})=[\min \{C I(B), C I(C)\}, \max \{C I(B), C I(C)\}] .
$$

Based on Eqs.(3) and (4), it is easy to see that both the CCI and the BCI do not implement the consistency degree of all the RPRs associated to an IVRPR, and as such might not reflect the consistency of an IVRPR accurately. This argument is used and exploited in the following sections to propose a new type of consistency measure for IVRPRs, which is called the average-case consistency measure of IVRPRs, and that is determined as the average consistency degree of all associated RPRs to the IVRPR. 


\section{Average-case Consistency Measure of IVRPRs}

This section proposes the average consistency index (ACI) of IVRPRs, followed by numerical examples and a comparative study to justify the feasibility of the new ACI to measure consistency of IVRPRs.

\subsection{Average Consistency Index of IVRPRs}

Let $\tilde{V}=\left(\left[v_{i j}^{-}, v_{i j}^{+}\right]\right)_{n \times n}$ be an IVRPR. The underlying idea of the new proposed average-case consistency measure consists in measuring the consistency degree of an IVRPR using the average consistency of all its associated RPRs. Indeed, associated RPRs of $\tilde{V}$ can be represented by $F=$ $\left(f_{i j}\right)_{n \times n} \in N_{\tilde{V}}$, and $f_{i j}(i<j)$ can be considered as a random variable taking values in $\left[v_{i j}^{-}, v_{i j}^{+}\right]$. These assumptions are used to propose the following formal definition of the ACI of the IVRPR $\tilde{V}$ :

Definition 4 (IVRPR Average Consistency Index). Let $\tilde{V}=\left(\left[v_{i j}^{-}, v_{i j}^{+}\right]\right)_{n \times n}$ be an IVRPR. The average consistency index (ACI) of $\tilde{V}$ is

$$
A C I(\tilde{V})=E(C I(F))
$$

where $F=\left(f_{i j}\right)_{n \times n} \in N_{\tilde{V}}$ is the random RPR associated to $\tilde{V}$, i.e. $f_{i j}$ are random variables in $\left[v_{i j}^{-}, v_{i j}^{+}\right]$ such that $f_{j i}=1-f_{i j} \forall i<j \in\{1,2, \ldots, n\} ; C I(F)$ is the consistency index random variable obtained via expression (2); and $E(C I(F))$ is the expected value of $C I(F)$. Consequently, we have

$$
A C I(\tilde{V})=1-\frac{4}{n \cdot(n-1) \cdot(n-2)} \sum_{i=1}^{n} \sum_{j=i+1}^{n} \sum_{k=j+1}^{n} E\left(\left|f_{i j}+f_{j k}-f_{i k}-0.5\right|\right) .
$$

The value $A C I(\tilde{V})$ measures on average the consistency degree of all the RPRs associated to $\tilde{V}$. Thus, the larger the value of $A C I(\tilde{V})$, the more consistent $\tilde{V}$ is.

The normal distribution is one of the most widely used probability distributions [30]. When a random variable $X$ is distributed normally with mean $u$ and variance $\sigma^{2}$, it is denoted by $X \sim N\left(u, \sigma^{2}\right)$ and its density function is:

$$
f(x)=\frac{1}{\sigma \sqrt{2 \pi}} \cdot e^{-\frac{(x-u)^{2}}{2 \sigma^{2}}} ; \quad-\infty<x<\infty
$$

In this paper, we assume that

$$
f_{i j} \sim N\left(u_{i j}, \sigma_{i j}^{2}\right)
$$

where

$$
u_{i j}=\frac{v_{i j}^{-}+v_{i j}^{+}}{2}
$$

and

$$
\sigma_{i j}=\frac{v_{i j}^{+}-v_{i j}^{-}}{6}
$$

These assumptions (Eqs. (8)-(10)) are based on the following reasons: 
(1) Based on Jong [16] and Dong et al. [10], decision makers often have certain consistency tendency in making pairwise comparisons, so in what follows it is assumed that $f_{i j}(i<j)$ relatively centralizes the domain close to $\frac{v_{i j}^{-}+v_{i j}^{+}}{2}$ and has a normal distribution, i.e., it is assumed that $f_{i j} \sim N\left(u_{i j}, \sigma_{i j}^{2}\right)$, where $u_{i j}=\frac{v_{i j}^{-}+v_{i j}^{+}}{2} ;$

(2) The probability of $f_{i j}$ distributed in the interval $\left[v_{i j}^{-}, v_{i j}^{+}\right]$should be close to 1 . According to the $3 \sigma$ principle of normally distributed variables [30], it is known that $P\left(u_{i j}-3 \sigma_{i j} \leq f_{i j} \leq u_{i j}+3 \sigma_{i j}\right) \approx 1$. Because $u_{i j}=\frac{v_{i j}^{-}+v_{i j}^{+}}{2}, u_{i j}-3 \sigma_{i j}=v_{i j}^{-}$and $u_{i j}+3 \sigma_{i j}=v_{i j}^{+}$, consequently, it is $\sigma_{i j}=\frac{v_{i j}^{+}-v_{i j}^{-}}{6}$.

The following result derives from the well known statistical result regarding independent and identically distributed random variables applied to the normal distribution type [30]:

Lemma 1. Let $X \sim N\left(u_{X}, \sigma_{X}^{2}\right), Y \sim N\left(u_{Y}, \sigma_{Y}^{2}\right)$ and $R \sim N\left(u_{R}, \sigma_{R}^{2}\right)$ be independent, and $Z=$ $X+Y-R-0.5$. Then it is $Z \sim N\left(u_{Z}, \sigma_{Z}^{2}\right)$, where $u_{Z}=u_{X}+u_{Y}-u_{R}-0.5$ and $\sigma_{Z}^{2}=\sigma_{X}^{2}+\sigma_{Y}^{2}+\sigma_{R}^{2}$.

According to Lemma 1 and the assumption, $f_{i j} \sim N\left(\frac{v_{i j}^{-}+v_{i j}^{+}}{2},\left(\frac{v_{i j}^{+}-v_{i j}^{-}}{6}\right)^{2}\right)$, we have the following main result, which provides the analytical procedure to compute the ACI of an IVRPR $\tilde{V}=\left(\tilde{v}_{i j}\right)_{n \times n}:$

Theorem 1. Let $\tilde{V}=\left(\left[v_{i j}^{-}, v_{i j}^{+}\right]\right)_{n \times n}$ be an IVRPR. Let

$$
\begin{gathered}
f_{i j} \sim N\left(\frac{v_{i j}^{-}+v_{i j}^{+}}{2},\left(\frac{v_{i j}^{+}-v_{i j}^{-}}{6}\right)^{2}\right) ; \\
\mu_{i j k}=\frac{v_{i j}^{-}+v_{i j}^{+}+v_{j k}^{-}+v_{j k}^{+}-v_{i k}^{-}-v_{i k}^{+}-1}{2} ; \\
\sigma_{i j k}=\frac{\sqrt{\left(v_{i j}^{+}-v_{i j}^{-}\right)^{2}+\left(v_{j k}^{+}-v_{j k}^{-}\right)^{2}+\left(v_{i k}^{+}-v_{i k}^{-}\right)^{2}}}{6} ;
\end{gathered}
$$

and $\Phi$ be the cumulative distribution function of the standard normal distribution $N(0,1)$. Then, the ACI of $\tilde{V}$ is expressed as follows:

$$
A C I(\tilde{V})=1-\frac{4}{n(n-1)(n-2)} \sum_{i=1}^{n} \sum_{j=i+1}^{n} \sum_{k=j+1}^{n} A C I_{i j k}
$$

where

$$
A C I_{i j k}= \begin{cases}\frac{2 \sigma_{i j k}}{\sqrt{2 \pi}}\left(e^{\left.-\frac{\mu_{i j k}^{2}}{2 \sigma_{i j k}^{2}}-e^{\frac{-9}{2}}\right)+\mu_{i j k}\left(1-2 \Phi\left(\frac{-\mu_{i j k}}{\sigma_{i j k}}\right)\right),}\right. & \text { If }-3 \leq \frac{-\mu_{i j k}}{\sigma_{i j k}} \leq 3 \\ \mu_{i j k}(\Phi(3)-\Phi(-3)), & \text { If } \frac{-\mu_{i j k}}{\sigma_{i j k}} \leq-3 \\ \mu_{i j k}(\Phi(-3)-\Phi(3)) . & \text { If } \frac{-\mu_{i j k}}{\sigma_{i j k}} \geq 3\end{cases}
$$


Proof. Let us denote $x=f_{i j}+f_{j k}-f_{i k}-0.5$. From Lemma 1 , it is

$$
x \sim N\left(\mu_{i j k}, \sigma_{i j k}^{2}\right)
$$

with $\mu_{i j k}=\frac{v_{i j}^{-}+v_{i j}^{+}+v_{j k}^{-}+v_{j k}^{+}-v_{i k}^{-}-v_{i k}^{+}-1}{2}$ and $\sigma_{i j k}=\frac{\sqrt{\left(v_{i j}^{+}-v_{i j}^{-}\right)^{2}+\left(v_{j k}^{+}-v_{j k}^{-}\right)^{2}+\left(v_{i k}^{+}-v_{i k}^{-}\right)^{2}}}{6}$.

According to Eq.(6), it is

$$
A C I(\tilde{V})=1-\frac{4}{n(n-1)(n-2)} \sum_{i=1}^{n} \sum_{j=i+1}^{n} \sum_{k=j+1}^{n} E\left(\left|f_{i j}+f_{j k}-f_{i k}-0.5\right|\right)
$$

and therefore it would be

$$
A C I(\tilde{V})=1-\frac{4}{n(n-1)(n-2)} \sum_{i=1}^{n} \sum_{j=i+1}^{n} \sum_{k=j+1}^{n} \frac{1}{\sigma_{i j k} \sqrt{2 \pi}} \int_{\mu_{i j k}-3 \sigma_{i j k}}^{\mu_{i j k}+3 \sigma_{i j k}}|x| e^{-\frac{\left(x-\mu_{i j k}\right)^{2}}{2 \sigma_{i j k}^{2}}} d x
$$

Let $y=\frac{x-\mu_{i j k}}{\sigma_{i j k}}$, then

$$
A C I(\tilde{V})=1-\frac{4}{n(n-1)(n-2)} \sum_{i=1}^{n} \sum_{j=i+1}^{n} \sum_{k=j+1}^{n} \frac{1}{\sqrt{2 \pi}} \int_{-3}^{3}\left|y \sigma_{i j k}+\mu_{i j k}\right| e^{-\frac{y^{2}}{2}} d y
$$

We have:

$$
\left|y \sigma_{i j k}+\mu_{i j k}\right|= \begin{cases}y \sigma_{i j k}+\mu_{i j k}, & y \geq \frac{-\mu_{i j k}}{\sigma_{i j k}} \\ -y \sigma_{i j k}-\mu_{i j k}, & y<\frac{-\mu_{i j k}}{\sigma_{i j k}}\end{cases}
$$

- If $-3 \leq \frac{-\mu_{i j k}}{\sigma_{i j k}} \leq 3$ :

$$
\begin{aligned}
& A C I_{i j k}=\frac{1}{\sqrt{2 \pi}} \int_{-3}^{3}\left|y \sigma_{i j k}+\mu_{i j k}\right| e^{-\frac{y^{2}}{2}} d y=\frac{1}{\sqrt{2 \pi}} \int_{-3}^{\frac{-\mu_{i j k}}{\sigma_{i j k}}}\left(-y \sigma_{i j k}-\mu_{i j k}\right) e^{-\frac{y^{2}}{2}} d y \\
& +\frac{1}{\sqrt{2 \pi}} \int_{\frac{-\mu_{i j k}}{\sigma_{i j k}}}^{3}\left(y \sigma_{i j k}+\mu_{i j k}\right) e^{-\frac{y^{2}}{2}} d y \\
& =\frac{\sigma_{i j k}}{\sqrt{2 \pi}} \int_{-3}^{\frac{-\mu_{i j k}}{\sigma_{i j k}}}-y e^{-\frac{y^{2}}{2}} d y-\mu_{i j k} \frac{1}{\sqrt{2 \pi}} \int_{-3}^{\frac{-\mu_{i j k}}{\sigma_{i j k}}} e^{-\frac{y^{2}}{2}} d y \\
& +\frac{-\sigma_{i j k}}{\sqrt{2 \pi}} \int_{\frac{-\mu_{i j k}}{\sigma_{i j k}}}^{3}-y e^{-\frac{y^{2}}{2}} d y+\mu_{i j k} \frac{1}{\sqrt{2 \pi}} \int_{\frac{-\mu_{i j k}}{\sigma_{i j k}}}^{3} e^{-\frac{y^{2}}{2}} d y \\
& =\frac{\sigma_{i j k}}{\sqrt{2 \pi}}\left(e^{-\frac{y^{2}}{2}}\right)_{-3}^{\frac{-\mu_{i j k}}{\sigma_{i j k}}}-\mu_{i j k}\left(\Phi\left(\frac{-\mu_{i j k}}{\sigma_{i j k}}\right)-\Phi(-3)\right) \\
& +\frac{-\sigma_{i j k}}{\sqrt{2 \pi}}\left(e^{-\frac{y^{2}}{2}}\right)_{\frac{-\mu_{i j k}}{\sigma_{i j k}}}^{3}+\mu_{i j k}\left(\Phi(3)-\Phi\left(\frac{-\mu_{i j k}}{\sigma_{i j k}}\right)\right) \\
& =\frac{2 \sigma_{i j k}}{\sqrt{2 \pi}}\left(e^{-\frac{\mu_{i j k}^{2}}{2 \sigma_{i j k}^{2}}}-e^{\frac{-9}{2}}\right)+\mu_{i j k}\left(1-2 \Phi\left(\frac{-\mu_{i j k}}{\sigma_{i j k}}\right)\right)
\end{aligned}
$$


- If $\frac{-\mu_{i j k}}{\sigma_{i j k}} \leq-3$ :

$$
\begin{aligned}
A C I_{i j k}=\frac{1}{\sqrt{2 \pi}} \int_{-3}^{3}\left|y \sigma_{i j k}+\mu_{i j k}\right| e^{-\frac{y^{2}}{2}} d y & =\frac{1}{\sqrt{2 \pi}} \int_{-3}^{3}\left(y \sigma_{i j k}+\mu_{i j k}\right) e^{-\frac{y^{2}}{2}} d y \\
& =\frac{-\sigma_{i j k}}{\sqrt{2 \pi}} \int_{-3}^{3}-y e^{-\frac{y^{2}}{2}} d y+\mu_{i j k} \frac{1}{\sqrt{2 \pi}} \int_{-3}^{3} e^{-\frac{y^{2}}{2}} d y \\
& =\frac{-\sigma_{i j k}}{\sqrt{2 \pi}}\left(e^{-\frac{y^{2}}{2}}\right)_{-3}^{3}+\mu_{i j k}(\Phi(3)-\Phi(-3)) \\
& =\mu_{i j k}(\Phi(3)-\Phi(-3))
\end{aligned}
$$

- If $\frac{-\mu_{i j k}}{\sigma_{i j k}} \geq 3$ :

$$
\begin{aligned}
A C I_{i j k}=\frac{1}{\sqrt{2 \pi}} \int_{-3}^{3}\left|y \sigma_{i j k}+\mu_{i j k}\right| e^{-\frac{y^{2}}{2}} d y & =\frac{1}{\sqrt{2 \pi}} \int_{-3}^{3}\left(-y \sigma_{i j k}-\mu_{i j k}\right) e^{-\frac{y^{2}}{2}} d y \\
& =\frac{\sigma_{i j k}}{\sqrt{2 \pi}} \int_{-3}^{3}-y e^{-\frac{y^{2}}{2}} d y-\mu_{i j k} \frac{1}{\sqrt{2 \pi}} \int_{-3}^{3} e^{-\frac{y^{2}}{2}} d y \\
& =\frac{\sigma_{i j k}}{\sqrt{2 \pi}}\left(e^{-\frac{y^{2}}{2}}\right)_{-3}^{3}-\mu_{i j k}(\Phi(3)-\Phi(-3)) \\
& =\mu_{i j k}(\Phi(-3)-\Phi(3))
\end{aligned}
$$

Therefore, $A C I(\tilde{V})=1-\frac{4}{n(n-1)(n-2)} \sum_{i=1}^{n} \sum_{j=i+1}^{n} \sum_{k=j+1}^{n} A C I_{i j k}$ and

$$
A C I_{i j k}= \begin{cases}\frac{2 \sigma_{i j k}}{\sqrt{2 \pi}}\left(e^{-\frac{\mu_{i j k}^{2}}{2 \sigma_{i j k}^{2}}-e^{\frac{-9}{2}}}\right)+\mu_{i j k}\left(1-2 \Phi\left(\frac{-\mu_{i j k}}{\sigma_{i j k}}\right)\right), & \text { If }-3 \leq \frac{-\mu_{i j k}}{\sigma_{i j k}} \leq 3 \\ \mu_{i j k}(\Phi(3)-\Phi(-3)), & \text { If } \frac{-\mu_{i j k}}{\sigma_{i j k}} \leq-3 \\ \mu_{i j k}(\Phi(-3)-\Phi(3)) . & \text { If } \frac{-\mu_{i j k}}{\sigma_{i j k}} \geq 3\end{cases}
$$

This completes the proof.

Corollary 1. Let $\tilde{V}$ be an IVRPR. Then, (1) $A C I(\tilde{V}) \in[0,1]$, and (2) $A C I(\tilde{V})=1$ if and only if $\tilde{V}$ is an $\operatorname{RPR}$ and $C C I(\tilde{V})=1$.

Proof. Because the average-case consistency index of $\tilde{V}$ is determined as the average consistency degree of all reciprocal preference relations associated to $\tilde{V}, A C I(\tilde{V}) \in[0,1]$. In the following, we prove (2).

- Sufficiency. Suppose that $\tilde{V}$ is an $\operatorname{RPR}$ and $C C I(\tilde{V})=1$. First, because $\tilde{V}$ is an RPR it is $C C I(\tilde{V})=A C I(\tilde{V})=C I(\tilde{V})$. Second, because $C C I(\tilde{V})=1$ it is also $A C I(\tilde{V})=1$. 
- Necessity. Suppose that $A C I(\tilde{V})=1$. Using reductio ad absurdum, without loss of generality, let us assume that $v_{12}^{-}<v_{12}^{+}$. Let $F=\left(f_{i j}\right)_{n \times n} \in N_{\tilde{V}}$ and $C I(F)=1$. Then, we can construct a new RPR $F^{\prime}=\left(f_{i j}^{\prime}\right)_{n \times n}$, where, $f_{i j}^{\prime}=f_{i j}(i, j \neq 1,2)$ and $f_{12}^{\prime} \in\left\{x \mid x \in\left[v_{12}^{-}, v_{12}^{+}\right], x \neq f_{12}\right\}$. Based on Eq. (2), $C I\left(F^{\prime}\right) \neq 1$. As a result, it would also be $A C I(\tilde{V}) \neq 1$, which contradicts the initial assumption. Thus, it has to be $v_{i j}^{-}=v_{j i}^{+} \forall i, j$. Consequently, it would be $C C I(\tilde{V})=$ $A C I(\tilde{V})=1$.

This completes the proof.

While corollary 1 provides the range of the ACI of an IVRPR, Theorem 1 provides the analytical procedure to compute the actual ACI value of an IVRPR $\tilde{V}=\left(\tilde{v}_{i j}\right)_{n \times n}$. First, based on the preference values of $\tilde{V}$, we can get the values of $\mu_{i j k}$ and $\sigma_{i j k}(i, j, k=1,2, \ldots, n)$. Second, Eq. (11) and the cumulative distribution function of the standard normal distribution $\Phi$ allow the computation of the value of $A C I(\tilde{V})$.

\subsection{Numerical examples for consistency measurement}

This section provides numerical examples that illustrate the consistency measurement using CCI, BCI and ACI, respectively. Consider the following four IVRPRs:

$$
\begin{gathered}
\tilde{V}_{1}=\left(\begin{array}{cccc}
{[0.5,0.5]} & {[0.7,1]} & {[0.1,0.4]} & {[0.7,1]} \\
- & {[0.5,0.5]} & {[0.2,1]} & {[0.2,0.5]} \\
- & - & {[0.5,0.5]} & {[0.4,0.8]} \\
- & - & - & {[0.5,0.5]}
\end{array}\right) ; \tilde{V}_{2}=\left(\begin{array}{ccccc}
{[0.5,0.5]} & {[0.4,1]} & {[0.2,0.4]} & {[0,0.5]} \\
- & {[0.5,0.5]} & {[0.5,1]} & {[0.6,0.8]} \\
- & - & {[0.5,0.5]} & {[0.6,1]} \\
- & - & - & {[0.5,0.5]}
\end{array}\right) \\
\tilde{V}_{3}=\left(\begin{array}{cccc}
{[0.5,0.5]} & {[0,1]} & {[0.4,0.6]} & {[0.4,0.6]} \\
- & {[0.5,0.5]} & {[0.2,0.8]} & {[0.3,0.7]} \\
- & - & {[0.5,0.5]} & {[0.3,0.7]} \\
- & - & - & {[0.5,0.5]}
\end{array}\right) ; \tilde{V}_{4}=\left(\begin{array}{cccc}
{[0.5,0.5]} & {[0,0.6]} & {[0.1,0.2]} & {[0.3,0.4]} \\
- & {[0.5,0.5]} & {[0,0.8]} & {[0.3,0.7]} \\
- & - & {[0.5,0.5]} & {[0.3,0.7]} \\
- & - & - & {[0.5,0.5]}
\end{array}\right)
\end{gathered}
$$

Applying Eqs. (3) and (4) and Theorem 1 we derive the corresponding CCI, BCI and ACI values of the above four IVRPRs, which are given in Table 1 below:

Table 1: CCI, BCI and ACI values

\begin{tabular}{ccccc}
\hline & $\tilde{V}_{1}$ & $\tilde{V}_{2}$ & $\tilde{V}_{3}$ & $\tilde{V}_{4}$ \\
\hline CCI & 1 & 1 & 1 & 1 \\
BCI & {$[0.63,0.77]$} & {$[0.5,0.83]$} & {$[0.7,0.7]$} & {$[0.67,0.77]$} \\
ACI & 0.72 & 0.67 & 1 & 0.9545 \\
\hline
\end{tabular}




\subsection{Comparative Study}

From Table 1, the following differences between the three different consistency measures of IVRPRs are highlighted:

(1) All four IVRPRS are consistent according to the classical consistency measure (CCI). However, according to the average-case consistency measure (ACI), it is noticed that two of the four IVRPRs do not have a very high consistency degree, while the other two do have a very high consistent degree.

(2) According to the boundary consistency index (BCI), consistency degrees for the four IVRPRS are always below 0.83 and above 0.5 , which might not be considered very high.

These observations show that the average-case consistency (ACI) behaves differently to the classical consistency measure (CCI) and the boundary consistency measure (BCI). Looking in more detail the values of the IVRPRs, we observe the following further observations:

- From $\tilde{V}_{1}$, we observe the following:

(i) It can be claimed that $x_{1} \succ x_{2}$ because all possible preference values of alternative $x_{1}$ over alternative $x_{2}$ are above 0.7 .

(ii) It can be claimed that $x_{3} \succ x_{1}$ because all possible preference values of alternative $x_{1}$ over alternative $x_{3}$ are below 0.4 .

(iii) It can be claimed that $x_{1} \succ x_{4}$ because all possible preference values of alternative $x_{1}$ over alternative $x_{4}$ are above 0.7 .

(iv) It can be claimed that $x_{4} \succ x_{2}$ because all possible preference values of alternative $x_{2}$ over alternative $x_{4}$ are below 0.5 .

(v) It is not clear that $x_{3} \succ x_{4}$, however the possibility of being $x_{3} \succ x_{4}$ is higher than that of being $x_{4} \succ x_{3}$ as it deduces from the comparison of their respective intervalvalued preferences $[0.4,0.8]$ and $[0.2,0.6]$, respectively. Indeed, given two interval numbers $\tilde{a}_{1}=\left[a_{1}^{-}, a_{1}^{+}\right]$and $\tilde{a}_{2}=\left[a_{2}^{-}, a_{2}^{+}\right]$, the possibility degree (PD) up to which the ordering relation $\tilde{a}_{1} \succ \tilde{a}_{2}$ is [33]:

$$
P\left(\tilde{a}_{1} \succ \tilde{a}_{2}\right)=\max \left\{1-\max \left\{\frac{a_{2}^{+}-a_{1}^{-}}{a_{1}^{+}-a_{1}^{-}+a_{2}^{+}-a_{2}^{-}}, 0\right\}, 0\right\}
$$

In the case of comparing alternatives with interval-valued reciprocal preferences, expression (12) can be used to conclude whether an alternative, $x_{i}$, is preferred to another one, $x_{j}$, by directly comparing the preference values $\tilde{v}_{i j}=\left[v_{i j}^{-}, v_{i j}^{+}\right]$and $\tilde{v}_{j i}=\left[v_{j i}^{-}, v_{j i}^{+}\right]$:

$$
\begin{aligned}
& -x_{i} \succ x_{j} \Leftrightarrow P\left(\tilde{v}_{i j} \succ \tilde{v}_{j i}\right)>0.5 \\
& -x_{i} \sim x_{j} \Leftrightarrow P\left(\tilde{v}_{i j} \succ \tilde{v}_{j i}\right)=0.5
\end{aligned}
$$


Reciprocity of preferences, $\left[v_{j i}^{-}, v_{j i}^{+}\right]=\left[1-v_{i j}^{+}, 1-v_{i j}^{-}\right]$, reduces expression (12) to

$$
P\left(\tilde{v}_{i j} \succ \tilde{v}_{j i}\right)=\max \left\{1-\max \left\{\frac{0.5-v_{i j}^{-}}{v_{i j}^{+}-v_{i j}^{-}}, 0\right\}, 0\right\}
$$

In our case, we have $\tilde{v}_{34}=\left[v_{34}^{-}, v_{34}^{+}\right]=[0.4,0.8]$ and $\tilde{v}_{43}=\left[v_{43}^{-}, v_{43}^{+}\right]=[0.2,0.6]$ and therefore:

$$
P\left(\widetilde{v}_{34} \succ \widetilde{v}_{43}\right)=0.75 ; \quad P\left(\widetilde{v}_{43} \succ \widetilde{v}_{34}\right)=0.25
$$

Putting all these consideration together for $\tilde{V}_{1}$, we have: $x_{3} \succ x_{1} \succ x_{4} \succ x_{2}$. However, it is more possible that $x_{2} \succ x_{3}$ rather than $x_{3} \succ x_{2}$ because $P\left(\widetilde{v}_{23} \succ \widetilde{v}_{32}\right)=5 / 8 ; \quad P\left(\widetilde{v}_{32} \succ \widetilde{v}_{23}\right)=3 / 8$, and therefore this inconsistency of preferences of $\tilde{V}_{1}$ is not accurately captured by the CCI measure.

- From $\tilde{V}_{2}$, we observe the following: $x_{3} \succ x_{1}, x_{4} \succ x_{1}, x_{3} \succ x_{4}, x_{2} \succ x_{3}$ so we can conclude that: $x_{2} \succ x_{3} \succ x_{4} \succ x_{1}$. However, we have that $P\left(\widetilde{v}_{12} \succ \widetilde{v}_{21}\right)=1 / 6$ and therefore it is more possible that $x_{1} \succ x_{2}$. Again, CCI does not accurately represent the consistency of this IVRPR.

- From $\tilde{V}_{3}$, using possibility values it can be deduced that all four alternatives are equally preferred, which is not appropriately represented by BCI with a low consistency value of 0.7 .

- From $\tilde{V}_{4}$, possibility values lead to: $x_{4} \succ x_{1}$ and $x_{3} \succ x_{1}$ with maximum possibility value, while at the same time we have $x_{4} \sim x_{3}$. We also have $x_{2} \succ x_{1}$ with possibility value $\frac{5}{6}$ and $x_{3} \succ x_{2}$ with possibility value of $\frac{5}{8}$. Thus, preferences in this case are highly consistent with the ordering $x_{3} \sim x_{4} \succ x_{2} \succ x_{1}$, but not completely consistent as there is also the relation $x_{2} \sim x_{4}$. It is clear that the ACI value reflects this ordering relationships better than the BCI value (too low) and the CCI value (complete consistency), respectively.

In the following, we further analyse why the three different consistency measures for IVRPRs behave differently. According to Eq.(3), we have

$$
C C I\left(\tilde{V}_{i}\right)=\max _{F \in N_{\tilde{V}_{i}}} C I(F), \quad i=1,2,3,4 .
$$

Solving the model described by expression (13) yields the associated RPR, $A_{i} \in N_{\tilde{V}_{i}}$, that satisfies

$$
C C I\left(\tilde{V}_{i}\right)=C I\left(A_{i}\right), \quad i=1,2,3,4 .
$$

Similarly, we may define the worst consistency index (WCI) of the IVRPR, $\tilde{V}_{i}$, as follows:

$$
W C I\left(\tilde{V}_{i}\right)=\min _{F \in N_{\tilde{V}_{i}}} C I(F), \quad i=1,2,3,4
$$

Solving the model described by expression (15) yields the associated RPR, $D_{i} \in N_{\tilde{V}_{i}}$, that satisfies

$$
W C I\left(\tilde{V}_{i}\right)=C I\left(D_{i}\right), \quad i=1,2,3,4 .
$$




\begin{tabular}{|c|c|c|c|c|c|c|c|c|c|c|c|c|c|c|c|c|}
\hline & \multicolumn{4}{|c|}{$A_{i}$} & \multicolumn{4}{|c|}{$B_{i}$} & \multicolumn{4}{|c|}{$C_{i}$} & \multicolumn{4}{|c|}{$D_{i}$} \\
\hline \multirow{4}{*}{$\tilde{V}_{1}$} & 0.5 & 0.7 & 0.4 & 0.7 & 0.5 & 0.7 & 0.1 & 0.7 & 0.5 & 1 & 0.4 & 1 & 0.5 & 1 & 0.1 & 1 \\
\hline & - & 0.5 & 0.2 & 0.5 & - & 0.5 & 0.2 & 0.2 & - & 0.5 & 1 & 0.5 & - & 0.5 & 1 & 0.2 \\
\hline & - & - & 0.5 & 0.8 & - & - & 0.5 & 0.4 & - & - & 0.5 & 0.8 & - & - & 0.5 & 0.798 \\
\hline & $(-$ & - & - & 0.5 & - & - & - & 0.5 & (- & - & - & 0.5 & - & - & - & 0.5 \\
\hline \multirow{4}{*}{$\tilde{V}_{2}$} & 0.5 & 0.4 & 0.4 & 0.5 & 0.5 & 0.4 & 0.2 & 0 & 0.5 & 1 & 0.4 & 0.5 & 0.5 & 1 & 0.4 & 0 \\
\hline & - & 0.5 & 0.5 & 0.6 & - & 0.5 & 0.5 & 0.6 & - & 0.5 & 1 & 0.8 & - & 0.5 & 1 & 0.8 \\
\hline & - & - & 0.5 & 0.6 & - & - & 0.5 & 0.6 & - & - & 0.5 & 1 & - & - & 0.5 & 1 \\
\hline & $1-$ & - & - & 0.5 & - & - & - & 0.5 & (- & - & - & 0.5 & $1-$ & - & - & $0.5)$ \\
\hline \multirow{4}{*}{$\tilde{V}_{3}$} & 0.5 & 0.4 & 0.6 & 0.6 & 0.5 & 0 & 0.4 & 0.4 & 0.5 & 1 & 0.6 & 0.6 & 0.5 & 1 & 0.6 & $0.4)$ \\
\hline & - & 0.5 & 0.7 & 0.7 & - & 0.5 & 0.2 & 0.3 & - & 0.5 & 0.8 & 0.7 & - & 0.5 & 0.8 & 0.7 \\
\hline & - & - & 0.5 & 0.5 & - & - & 0.5 & 0.3 & - & - & 0.5 & 0.7 & - & - & 0.5 & 0.7 \\
\hline & $1-$ & - & - & 0.5 & - & - & - & 0.5 & $1-$ & - & - & 0.5 & (- & - & - & $0.5)$ \\
\hline \multirow{4}{*}{$\tilde{V}_{4}$} & 0.5 & 0.2 & 0.2 & $0.4)$ & 0.5 & 0 & 0.1 & $0.3)$ & 0.5 & 0.6 & 0.2 & $0.4)$ & $(0.5$ & 0 & 0.1 & $0.4)$ \\
\hline & - & 0.5 & 0.5 & 0.7 & - & 0.5 & 0 & 0.3 & - & 0.5 & 0.8 & 0.7 & - & 0.5 & 0 & 0.3 \\
\hline & - & - & 0.5 & 0.7 & - & - & 0.5 & 0.3 & - & - & 0.5 & 0.7 & - & - & 0.5 & 0.3 \\
\hline & - & - & - & 0.5 & - & - & - & 0.5 & - & - & - & 0.5 & - & - & - & 0.5 \\
\hline
\end{tabular}

Based on Eq.(1), we can easily get the boundary RPRs $B_{i}$ and $C_{i}$ associated to $\tilde{V}_{i}(i=1,2,3,4)$, and

$$
B C I\left(\tilde{V}_{i}\right)=\left[\min \left\{C I\left(B_{i}\right), C I\left(C_{i}\right)\right\}, \max \left\{C I\left(B_{i}\right), C I\left(C_{i}\right)\right\}\right] .
$$

Tables 2 and 3 provide the above associated RPRs, $A_{i}, B_{i}, C_{i}, D_{i}$, and their corresponding consistency values, respectively.

Clearly, the data in Tables 1-3 is in line with Eqs. (14), (16) and (17). According to Eqs. (13) and (14), the CCI of the IVRPR $\tilde{V}_{i}$ is determined by its associated RPR with highest consistency degree, $A_{i}$. Meanwhile, according to Eqs. (15) and (16), the WCI of the IVRPR $\tilde{V}_{i}$ is determined by its associated RPR with worst consistency degree, $D_{i}$. In other words, the CCI and WCI values are the upper and lower bounds for the consistency degree of IVRPRs, respectively. Moreover, based on Eq.(17), the BCI of $\tilde{V}_{i}$ is determined by its two associated boundary RPRs, $B_{i}$ and $C_{i}$, whose consistency values will obviously be bounded by the WCI and CCI values of $\tilde{V}_{i}$, i.e. $B C I\left(\tilde{V}_{i}\right) \in\left[W C I\left(\tilde{V}_{i}\right), C C I\left(\tilde{V}_{i}\right)\right]$. However, as Wang shown recently in [29], both $B_{i}$ and $C_{i}$ cannot provide reliable information to measure the consistency degree of $\tilde{V}_{i}$.

Different from the classical consistency measure and the boundary consistency measure, the ACI 
Table 3: Consistency degrees of Associated RPRs

\begin{tabular}{ccccc}
\hline & $A_{i}$ & $B_{i}$ & $C_{i}$ & $D_{i}$ \\
\hline$i=1$ & 1 & 0.77 & 0.63 & 0.43 \\
$i=2$ & 1 & 0.83 & 0.5 & 0.33 \\
$i=3$ & 1 & 0.7 & 0.7 & 0.63 \\
$i=4$ & 1 & 0.67 & 0.77 & 0.63 \\
\hline
\end{tabular}

of an IVRPR is determined using all the IVRPR associated RPRs' consistency values. Obviously, in this case the following also holds:

$$
A C I\left(\tilde{V}_{i}\right) \in\left[W C I\left(\tilde{V}_{i}\right), C C I\left(\tilde{V}_{i}\right)\right] .
$$

In summary, the CCI, the WCI and the ACI provide the upper bound, lower bound and average consistency degree of IVRPRs, respectively, and each one complements the other, making their combined use comprehensively reflect the consistency status of IVRPRs.

\section{Average-case consistency improving method}

For RPRs of unacceptable consistency, consistency improving methods [8-10, 36, 37] have been developed. In this section, an average-case consistency improving method with the aim of obtaining a modified IVRPR with a required ACI is developed.

\subsection{A method to improve ACI}

The basic idea of the proposed average-case consistency improving method is based on the concept of adjusted IVRPR of a given IVRPR and their relationship regarding their $C C I$ and $W C I$ values as per the following definition and results:

Definition 5 (Adjusted IVRPR). Let $\tilde{V}=\left(\left[v_{i j}^{-}, v_{i j}^{+}\right]\right)_{n \times n}$ be an IVRPR. $A=\left(a_{i j}\right)_{n \times n}$ the RPR associated to $\widetilde{V}$ with best consistency degree, i.e. $C I(A)=C C I(\tilde{V})$; and $D=\left(d_{i j}\right)_{n \times n}$ the RPR associated to $\widetilde{V}$ with worst consistency degree, i.e. $C I(D)=W C I(\tilde{V})$. The preference relation $\widetilde{V}^{\prime}=\left(\left[v_{i j}^{\prime-}, v_{i j}^{\prime+}\right]\right)_{n \times n}$, constructed according to the following three rules

R1: If $a_{i j}<d_{i j}$, then let $v_{i j}^{\prime-}=v_{i j}^{-}$and $v_{i j}^{\prime+} \in\left[a_{i j}, d_{i j}\right)$.

R2: If $a_{i j}>d_{i j}$, then let $v_{i j}^{\prime-} \in\left(d_{i j}, a_{i j}\right]$ and $v_{i j}^{\prime+}=v_{i j}^{+}$.

R3: If $a_{i j}=d_{i j}$, then let $v_{i j}^{\prime-}=v_{i j}^{-}$and $v_{i j}^{\prime+}=v_{i j}^{+}$.

is called the adjusted IVRPR associated to $\widetilde{V}$. 
Given an IVRPR with specific $W C I$ value, the following result allows to improve the $W C I$ value while, simultaneously, preserving the IVRPR $C C I$ value by computing the corresponding associated adjusted IVRPR.

Lemma 2. Let $\tilde{V}$ be an IVRPR and $\tilde{V}^{\prime}$ be its adjusted IVRPR. Then it is $C C I\left(\widetilde{V}^{\prime}\right)=C C I(\widetilde{V})$, and $W C I\left(\widetilde{V}^{\prime}\right) \geq W C I(\widetilde{V})$.

Proof. According to R1-R3, we have that $\left[v_{i j}^{\prime-}, v_{i j}^{\prime+}\right] \subset\left[v_{i j}^{-}, v_{i j}^{+}\right]$and therefore any RPR associated to $\widetilde{V}^{\prime}$ is also an RPR associated to $\widetilde{V}$. Also, R1-R3 imply that $a_{i j} \in\left[v_{i j}^{\prime-}, v_{i j}^{\prime+}\right]$ in the adjustment process, and consequently it is: $C C I\left(\widetilde{V}^{\prime}\right)=C C I(\widetilde{V})=C I(A)$. On the other hand, R1-R3 imply that $d_{i j} \notin\left[v_{i j}^{\prime-}, v_{i j}^{\prime+}\right]$, and it can be concluded that $W C I\left(\widetilde{V}^{\prime}\right) \geq W C I(\widetilde{V})=C I(D)$. This completes the proof.

A direct consequence of Lemma 2 is that an IVRPR $\widetilde{V}$ and its adjusted IVRPR $\widetilde{V}^{\prime}$ have the same CCI value.

Corollary 2. Let $\tilde{V}$ be an IVRPR and $\tilde{V}^{\prime}$ its adjusted IVRPR. If $A=\left(a_{i j}\right)_{n \times n}$ is the RPR associated to $\widetilde{V}$ with best consistency degree, i.e. $C I(A)=C C I(\tilde{V})$, then $C I(A)=C C I\left(\tilde{V}^{\prime}\right)$.

According to Eq.(18), $A C I$ is located between $W C I$ and $C C I$, and therefore it is feasible to develop an average-case consistency improving method to improve the $A C I$ of IVRPRs by increasing the value of $W C I$. To achieve this, a linear programming based method to improve the $C C I$ of IVRPRs is presented.

Linear programming based method to improve the CCI of IVRPRs. Let $\tilde{V}=\left(\left[v_{i j}^{-}, v_{i j}^{+}\right]\right)_{n \times n}$ be an IVRPR. The main aim when improving the CCI of $\widetilde{V}$ is to find a suitable IVRPR, $\widetilde{V}^{*}=\left(\left[v_{i j}^{*-}, v_{i j}^{*+}\right]\right)$, with $C C I\left(\widetilde{V}^{*}\right)=1$. To preserve the information in $\widetilde{V}$ as much as possible, $\widetilde{V}^{*}$ is chosen as the IVRPR closest to $\tilde{V}$. When the distance between two IVRPRs is computed using the Manhattan distance $[5,6]$, we have that $\widetilde{V}^{*}$ is the solution to the following linear programming problem:

$$
(L P-1)\left\{\begin{array}{l}
\min \frac{1}{n(n-1)} \sum_{i=1}^{n} \sum_{j=i+1}^{n}\left(\left|v_{i j}^{+}-v_{i j}^{*+}\right|+\left|v_{i j}^{-}-v_{i j}^{*-}\right|\right) \\
\text { s.t. } C C I\left(\widetilde{V}^{*}\right)=1
\end{array}\right.
$$

The below algorithm 1 provides a formal description of the proposed average-case consistency improving method. 
Input: The IVRPR $\widetilde{V}=\left(\widetilde{v}_{i j}\right)_{n \times n}$, and the average consistency threshold $\overline{A C I}$.

Output: The adjusted IVRPR $\widetilde{V}^{\prime}=\left(\widetilde{v}_{i j}^{\prime}\right)_{n \times n}$, and $A C I\left(\widetilde{V}^{\prime}\right)$.

Step1. Let $t=0$, and $\widetilde{V}^{t}=\widetilde{V}$.

Step 2. if $C C I\left(\widetilde{V}^{t}\right)<1$ then

apply $(L P-1)$ method to obtain new IVRPR $\widetilde{V}^{t *}$, such that $C C I\left(\widetilde{V}^{t *}\right)=1$.

Let $\widetilde{V}^{t}=\widetilde{V}^{t *}$ and go to Step 3.

else

go to Step 3.

end if

Step 3. Apply Theorem 1 to calculate $A C I\left(\widetilde{V}^{t}\right)$.

if $A C I\left(\widetilde{V}^{t}\right)<\overline{A C I}$ then

| go to Step 4.

else

| go to Step 5 .

end if

Step 4. Compute associated RPRs to $\widetilde{V}^{t}, A^{t}=\left(a_{i j}^{t}\right)_{n \times n}$ and $D^{t}=\left(d_{i j}^{t}\right)_{n \times n}$, such that:

$C I\left(A^{t}\right)=C C I\left(\tilde{V}^{t}\right)$ and $C I\left(D^{t}\right)=W C I\left(\tilde{V}^{t}\right)$. Apply R1-R3 from Definition 5 to compute the adjusted IVRPR, $\widetilde{V}^{t+1}$, associated to $\widetilde{V}^{t}$. Let $t=t+1$, and go to Step $\mathbf{3}$.

Step 5. $\tilde{V}^{\prime}=\tilde{V}^{t}$.

\section{Algorithm 1: IVRPR average-case consistency improving method.}

The following result proves that when the average consistency threshold $\overline{A C I}$ increases towards its maximum value 1 then the average-case consistency measure of the adjusted IVRPR $\widetilde{V}^{\prime}=\left(\widetilde{v}_{i j}^{\prime}\right)_{n \times n}$ derived from Algorithm 1, $A C I\left(\widetilde{V}^{\prime}\right)$, also increases towards its maximum value 1.

Theorem 2. Let $\tilde{V}$ be an IVRPR, and $\widetilde{V}^{\prime}$ the adjusted IVRPR derived from Algorithm 1 with an average consistency threshold $\overline{A C I}$. Then:

$$
\lim _{A C I \rightarrow 1} A C I\left(\widetilde{V}^{\prime}\right)=1
$$

Proof. Based on Definition 5, we have that $d_{i j}^{t+1}$ values obtained from Rule 1 are lower than the corresponding value $d_{i j}^{t}$ and greater than $a_{i j}$, while $d_{i j}^{t+1}$ values obtained from Rule 2 are greater than the corresponding value $d_{i j}^{t}$ and lower than $a_{i j}$, respectively. Values $d_{i j}^{t+1}$ obtained from Rule 3 are equal to the corresponding value $d_{i j}^{t}$ and also equal to $a_{i j}$. Thus the $\operatorname{RPR} D^{t+1}$ is closer to $A$ than RPR $D^{t}$. Furthermore, each element of $D^{t}$ can be classed as being in a strictly monotonic sequence bounded by the corresponding element of $A$, and therefore when Lemma 2 is repeatedly applied then we have that the sequence of RPRs $\left\{D^{t} ; t \in \mathbb{N}\right\}$ converges towards $A$. Therefore, when $\overline{A C I} \rightarrow 1$ we have that $D^{t} \rightarrow A$, and because $A C I(\tilde{V}) \in[W C I(\tilde{V}), C C I(\tilde{V})]$ it is

$$
\lim _{A C I \rightarrow 1} A C I\left(\widetilde{V}^{\prime}\right)=1
$$


This completes the proof.

Theorem 2 guarantees that the proposed average-case consistency improving method can transform any IVRPR into one with a required ACI.

The design of the consistency improving method is a classical topic in decision making with preference relations. Generally, the adjusted values should only be considered as a decision aid which decision makers use as a reference to modify his preference values. The proposed average-case consistency improving method follows this research line, and both (LP-1) and R1-R3 should be used as a reference for decision makers to improve the consistency level of IVRPRs.

\subsection{Numerical example for consistency improvement}

Next, we use the IVRPR $\widetilde{V}_{1}$ presented in Section 3.2 as an example to illustrate the use of the average-case consistency improving method. Without loss of generality, in this example, it is set $\overline{A C I}=0.9$.

Algorithm 1 - Iteration 1. Step 1. Let $\widetilde{V}_{1}^{0}=\widetilde{V}_{1}$. According to Table 3, we have $C C I\left(\widetilde{V}_{1}^{0}\right)=$ $C I\left(A_{1}^{0}\right)=1$, with $A_{1}^{0}$ given in Table 2

$$
A_{1}^{0}=\left(\begin{array}{cccc}
0.5 & 0.7 & 0.4 & 0.7 \\
- & 0.5 & 0.2 & 0.5 \\
- & - & 0.5 & 0.8 \\
- & - & - & 0.5
\end{array}\right)
$$

Go to Step 3. From Table 1, we have $A C I\left(\widetilde{V}_{1}^{0}\right)=0.72$, which is lower than $\overline{A C I}$. Go to Step 4. Solving Eq.(15), the associated RPR to $\widetilde{V}_{1}^{0}$ with worst consistency degree is

$$
D_{1}^{0}=\left(\begin{array}{cccc}
0.5 & 1 & 0.1 & 1 \\
- & 0.5 & 1 & 0.2 \\
- & - & 0.5 & 0.798 \\
- & - & - & 0.5
\end{array}\right)
$$

According to R1-R3 from Definition 5, the directions to improve the $A C I$ of $\widetilde{V}_{1}^{0}$ are:

(i) Increase the value of $v_{13}^{0,-}, v_{24}^{0,-}$ and $v_{34}^{0,-}$ :

$$
v_{13}^{-, 1} \in(0.1,0.4] ; v_{24}^{-, 1} \in(0.2,0.5] ; v_{34}^{-, 1} \in(0.798,0.8] .
$$

(ii) Decrease the value of $v_{12}^{0,+}, v_{14}^{0,+}$ and $v_{23}^{0,+}$ :

$$
v_{12}^{+, 1} \in[0.7,1) ; v_{14}^{+, 1} \in[0.7,1) ; v_{23}^{+, 1} \in[0.2,1) .
$$


Following the above suggestions and assuming, without loss of generality, that the new (adjusted) $\operatorname{IVRPR} \widetilde{V}_{1}^{1}=\left(\widetilde{v}_{i j}^{1}\right)$ given is

$$
\widetilde{V}_{1}^{1}=\left(\begin{array}{cccc}
{[0.5,0.5]} & {[0.7,0.8]} & {[0.2,0.4]} & {[0.7,0.9]} \\
- & {[0.5,0.5]} & {[0.2,0.8]} & {[0.3,0.5]} \\
- & - & {[0.5,0.5]} & {[0.799,0.8]} \\
- & - & - & {[0.5,0.5]}
\end{array}\right)
$$

From Corollary 2, it is $A_{1}^{1}=A_{1}^{0}$ and therefore $C C I\left(\widetilde{V}_{1}^{1}\right)=C I\left(A_{1}^{1}\right)=C I\left(A_{1}^{0}\right)=1$ and therefore go to Step 3 for a new iteration of Algorithm 1.

Algorithm 1 - Iteration 2. Step 3. Eq.(11) results in $A C I\left(\widetilde{V}_{1}^{1}\right)=0.8$, which is still below the threshold value $\overline{A C I}$. Go to Step 4. Solving Eq.(15), the associated RPR to $\widetilde{V}_{1}^{1}$ with worst consistency degree is

$$
D_{1}^{1}=\left(\begin{array}{cccc}
0.5 & 0.798 & 0.2 & 0.9 \\
- & 0.5 & 0.8 & 0.3 \\
- & - & 0.5 & 0.799 \\
- & - & - & 0.5
\end{array}\right)
$$

According to R1-R3 from Definition 5, the directions to improve the $A C I$ of $\widetilde{V}_{1}^{1}$ are:

(i) Increase the value of $v_{13}^{-, 1}, v_{24}^{-, 1}$ and $v_{34}^{-, 1}$ :

$$
v_{13}^{-, 2} \in(0.2,0.4] ; v_{24}^{-, 2} \in(0.3,0.5] ; v_{34}^{-, 2} \in(0.799,0.8] .
$$

(ii) Decrease the value of $v_{12}^{+, 1}, v_{14}^{+, 1}$ and $v_{23}^{+, 1}$ :

$$
v_{12}^{+, 2} \in[0.7,0.798) ; v_{14}^{+, 2} \in[0.7,0.9) ; v_{23}^{+, 2} \in[0.2,0.8) .
$$

Following the above suggestions and assuming, without loss of generality, that the new (adjusted) $\operatorname{IVRPR} \widetilde{V}_{1}^{2}=\left(\widetilde{v}_{i j}^{2}\right)$ is

$$
\widetilde{V}_{1}^{2}=\left(\begin{array}{cccc}
{[0.5,0.5]} & {[0.7,0.75]} & {[0.3,0.4]} & {[0.7,0.8]} \\
- & {[0.5,0.5]} & {[0.2,0.5]} & {[0.4,0.5]} \\
- & - & {[0.5,0.5]} & {[0.8,0.8]} \\
- & - & - & {[0.5,0.5]}
\end{array}\right)
$$

A new iteration of Algorithm 1 is carried out.

Algorithm 1 - Iteration 3. Step 3. Because $A C I\left(\widetilde{V}_{1}^{2}\right)=0.9$, the threshold value $\overline{A C I}$ has been reached. Go to Step 5, which ends the algorithm and returns as outputs:

$$
\widetilde{V}^{\prime}=\widetilde{V}_{1}^{2} ; \quad A C I\left(\widetilde{V}^{\prime}\right)=0.9
$$




\subsection{Simulation experiments}

In this subsection, we explore the average-case consistency improving method by means of simulation experiments. Let $\widetilde{V}, \widetilde{V}^{\prime}, A$ and $D$ as per Definition 5 . In order to show the process to improve the values $C C I, W C I$ and $A C I$ of $\widetilde{V}$ when applying the presented average-case consistency improving method, a parameter $\alpha(0<\alpha<1)$ is introduced to automatically revise the preference values in $\widetilde{V}$ to derive its adjusted IVRPR $\widetilde{V}^{\prime}$. To do so, R1-R3 in Definition 5 are re-defined as follows:

R1': If $a_{i j}<d_{i j}$, then let $v_{i j}^{\prime-}=v_{i j}^{-}$and $v_{i j}^{\prime+}=\max \left\{a_{i j}, d_{i j}-\alpha\right\}$.

R2': If $a_{i j}>d_{i j}$, then let $v_{i j}^{\prime-}=\min \left\{d_{i j}+\alpha, a_{i j}\right\}$ and $v_{i j}^{++}=v_{i j}^{+}$.

R3': If $a_{i j}=d_{i j}$, then let $v_{i j}^{\prime-}=v_{i j}^{-}$and $v_{i j}^{\prime+}=v_{i j}^{+}$.

The larger the parameter $\alpha$ value is, the larger the adjustment amount will be in R1'-R3'. In Algorithm 1, we replace R1-R3 with R1'-R3', respectively, and then obtain an automatic version of Algorithm 1, that we will refer to as Algorithm 2. Next, we set different $\alpha$ values, and run Algorithm 2 to improve the consistency indexes $(C C I, W C I$ and $A C I)$ of IVRPRs. Because $A C I\left(\widetilde{V}_{3}\right)=1$ and $A C I\left(\widetilde{V}_{4}\right)=0.9545$ are already quite high, we replace them with two new IVRPRs, $\widetilde{V}_{5}$ and $\widetilde{V}_{6}$, taken from [35] and [28], respectively:

$\widetilde{V}_{5}=\left(\begin{array}{ccccc}{[0.5,0.5]} & {[0.3,0.4]} & {[0.5,0.7]} & {[0.4,0.5]} \\ - & {[0.5,0.5]} & {[0.6,0.8]} & {[0.2,0.6]} \\ - & - & {[0.5,0.5]} & {[0.4,0.8]} \\ - & - & - & {[0.5,0.5]}\end{array}\right) ; \widetilde{V}_{6}=\left(\begin{array}{ccccc}{[0.5,0.5]} & {[0.75,0.85]} & {[0.65,0.75]} & {[0.35,0.45]} \\ - & {[0.5,0.5]} & {[0.5,0.65]} & {[0.5,0.65]} \\ - & - & {[0.5,0.5]} & {[0.62,0.75]} \\ - & - & - & {[0.5,0.5]}\end{array}\right)$.

Algorithm 2 is applied to $\widetilde{V}_{1}, \widetilde{V}_{2}, \widetilde{V}_{5}$ and $\widetilde{V}_{6}$ with three different $\alpha$ values of $0.05,0.1$ and 0.15 . The improving process of the consistency indexes for each IVRPR is illustrated in Figures 1-4, respectively. The following observations can be drawn:

(1) Notice that $C C I$ if not 1 at iteration 1 of Algorithm 2, then it is set to 1 from iteration 2 and remains as such thereafter.

(2) Both the $A C I$ and $W C I$ values of $\widetilde{V}_{i}(i=1,2,5,6)$ increase in each iteration. This was already proved theoretically in Lemma 2 and Theorem 2, respectively. This implies that both the ACI and $W C I$ can be improved by using the average-case consistency improving method, which further justifies the feasibility of our proposal.

(3) It will take less iterations to reach an established consistency index the larger the $\alpha$ value is. Indeed, the larger the value of $\alpha$ the closer the $\operatorname{RPR} D$ is to the $\operatorname{RPR} A$, and consequently the $A C I$ value will be pushed closer to the $C C I$ value, which is set to its maximum value of 1 in the first iteration of the proposed algorithm. 


\section{Conclusion}

In this paper, the average-case consistency measure of IVRPRs has been proposed, analysed and compared against the two existing consistency measures of IVRPR: the classical consistency measure and the boundary consistency measure. The underlying idea of the average consistency measure consist in measuring the consistency degree of the IVRPR using the average of the consistency degrees of all its associated RPRs. The internal mechanisms of the different consistency measures have been analysed, and the combined use of the classical consistency measure, the worst consistency measure and the average consistency measure are proposed to comprehensively reflect the consistency status of IVRPRs. Furthermore, an average-case consistency improving method aimed to obtain a modified IVRPR with a required ACI is proposed, theoretically justified and corroborated via an experiment simulation. This consistency improvement method relies on the concept of adjusted IVRPR associated to a given IVRPR and on the application of linear programming based method to improve the classical consistency index of an IVRPR.

In future, the following issues need attention:

(1) The consistency measure has been used as a driver to estimate missing information in incomplete RPRs [15]. In future, it will be interesting to study a method based on the ACI to estimate the missing values of incomplete IVRPRs, and to compare it with existing approaches based on the CCI, as the ones reported in $[25,26,31,32]$.

(2) The proposed average-case consistency measure of IVRPRs is based on the additive transitivity. Since the multiplicative transitivity is an alternative approach to measure consistency of RPRs [4], it will be interesting to study the mathematical properties of a corresponding average multiplicative consistency of IVRPRs.

(3) Establishing the consistency threshold $\overline{C I}$ for RPRs is a very challenging task, and is still open. It will be necessary to provide a systematic investigation to establish the thresholds for the consistency indexes CI, CCI, WCI and ACI.

\section{Acknowledgements}

This work was supported by the grants (Nos. 71171160 and 71571124) from NSF of China, the grant (No. skqy201606) from Sichuan University, the grant (No. TIN2013-40658-P) from the FEDER funds, and the grant (No. TIC-5991) from the Andalusian Excellence Project.

\section{References}

[1] S. Alonso, F. Chiclana, F. Herrera, E. Herrera-Viedma, J. Alcalá-Fdez, C. Porcel, A consistencybased procedure to estimate missing pairwise preference values. International Journal of Intelligent Systems 23(2) (2008) 155-175. 
[2] B.D. Baets, H.D. Meyer, B.D. Schuymer, S. Jenei, Cyclic evaluation of transitivity of reciprocal relations. Social Choice and Welfare 26(2) (2006) 217-238.

[3] F. Chiclana, F. Herrera, E. Herrera-Viedma, Integrating multiplicative preference relations in a multipurpose decision-making model based on fuzzy preference relations. Fuzzy Sets and Systems $122(2001) 277-291$.

[4] F. Chiclana, E. Herrera-Viedma, S. Alonso, F. Herrera, Cardinal consistency of reciprocal preference relations: A Characterization of multiplicative transitivity. IEEE Transactions on Fuzzy Systems 17(1) (2009) 14-23.

[5] F. Chiclana, J. M. Tapia García, M. J. del Moral, E. Herrera-Viedma, A statistical comparative study of different similarity measures of consensus in group decision making. Information Sciences, 221:110-123, 2013.

[6] M. M. Deza, E. Deza. Encyclopedia of Distances. Springer Berlin Heidelberg, 2009.

[7] Y.C Dong, E. Herrera-Viedma, Consistency-driven automatic methodology to set interval numerical scales of 2-tuple linguistic term sets and its use in the linguistic GDM with preference relation, IEEE Transactions on Cybernetics 45 (2015) 780-792.

[8] Y.C. Dong, X. Chen, C.C. Li, W.C. Hong, Y.F. Xu, Consistency issues of interval pairwise comparison matrices. Soft Computing 19 (2015) 2321-2335.

[9] Y.C. Dong, C.C. Li, F. Herrera, An optimization-based approach to adjusting unbalanced linguistic preference relations to obtain a required consistency level. Information Sciences 292(5) (2015) 27-38.

[10] Y.C. Dong, Y.F. Xu, H.Y. Li, On consistency measures of linguistic preference relations. European Journal of Operational Research 189(2) (2008) 430-444.

[11] Y.C. Dong, H.J. Zhang, E. Herrera-Viedma, Integrating experts' weights generated dynamically into the consensus reaching process and its applications in managing non-cooperative behaviors. Decision Support Systems 84(2016) 1-15.

[12] M. Fedrizzi, R.A.M. Pereira, Positive fuzzy matrices, dominant eigenvalues and an extension of Saaty's analytical hierarchy process. in Proceeding of 6th World Congress of International Fuzzy Systems Association (IFSA) São Paulo, Brazil, 245-247, 1995.

[13] J. Fodor, M. Roubens, Fuzzy preference modelling and multicriteria decision support. Kluwer, Dordrecht, 1994. 
[14] E. Herrera-Viedma, F. Herrera, F. Chiclana, M. Luque, Some issues on consistency of fuzzy preference relations. European Journal of Operational Research 154(1) (2004) 98-109.

[15] E. Herrera-Viedma, F. Chiclana, F. Herrera, S. Alonso, Group decision making model with incomplete fuzzy preference relations based on additive consistency. IEEE Transactions on Systems, Man and Cybernetics Part B: Cybernetics 37(1) (2007) 176-189.

[16] P. Jong, A statistical approach to Saaty's scaling method for priorities. Journal of Mathematical Psychology 28 (1984) 467-478.

[17] W. Koczkodaj, R. Szwarc, On axiomatization of inconsistency indicators in pairwise comparisons. Fundamenta Informaticae 132(4) (2014) 485-500.

[18] W. Koczkodaj, A new definition of consistency of pairwise comparisons. Mathematical and Computer Modelling 18(7) (1993) 79-84.

[19] F. Liu, Acceptable consistency analysis of interval reciprocal comparison matrices. Fuzzy Sets and Systems 160 (2009) 2686-2700.

[20] F. Liu, W.G. Zhang, Z.X. Wang, A goal programming model for incomplete interval multiplicative preference relations and its application in group decision-making. European Journal of Operational Research 218 (2012) 747-754.

[21] T.L. Saaty, The Analytic Hierarchy Process. McGraw-Hill, New York, 1980.

[22] B. Srdjevic, Combining different prioritization methods in analytic hierarchy process synthesis. Computers $\& 3$ Operations Research 32 (2005) 1897-1919.

[23] B. Srdjevic, Z. Srdjevic, Synthesis of individual best local priority vectors in AHP-group decision making. Applied Soft Computing 13(4) (2013) 2045-2056.

[24] T. Tanino, Fuzzy preference relations in group decision making, in: J. Kacprzyk, M. Roubens (Eds.), Non-Conventional Preference Relations in Decision Making, Springer Berlin Heidelberg 301 (1988) 54-71.

[25] R. Ureña, F. Chiclana, J.A. Morente, E. Herrera-Viedma, Managing incomplete preference relations in decision making: A review and future trends. Information Sciences 302 (2015) 14-32.

[26] R. Ureña, F. Chiclana, H. Fujita, E. Herrera-Viedma, Confidence-consistency driven group decision making approach with incomplete reciprocal intuitionistic preference relations. KnowledgeBased Systems 89 (2015) 86-96.

[27] Y.M. Wang, J.B. Yang, D.L. Xu, Interval weight generation approaches based on consistency test and interval comparison matrices. Applied Mathematics and Computation 167(1) (2005) 252-273. 
[28] Z.-J. Wang, K.W. Li, Goal programming approaches to deriving interval weights based on interval fuzzy preference relations. Information Sciences 193 (2012) 180-198.

[29] Z.-J. Wang, A note on A goal programming model for incomplete interval multiplicative preference relations and its application in group decision-making. European Journal of Operational Research $247(3)(2015) 867-871$.

[30] L. Wasseman, All of Statistics. Springer-Verlag, 2004.

[31] J. Wu, F. Chiclana, A social network analysis trust-consensus based approach to group decisionmaking problems with interval-valued fuzzy reciprocal preference relations. Knowledge-Based Systems 59 (2014) 97-107.

[32] J. Wu, F. Chiclana, Multiplicative consistency of intuitionistic reciprocal preference relations and its application to missing values estimation and consensus building. Knowledge-Based Systems 71 (2014) 187-200.

[33] J. Wu, F. Chiclana, A risk attitudinal ranking method for interval-valued intuitionistic fuzzy numbers based on novel score and accuracy expected functions. Applied Soft Computing 22 (2014) $272-286$.

[34] J. Wu, R. Xiong, F. Chiclana, Uninorm trust propagation and aggregation methods for group decision making in social network with four tuple information. Knowledge-Based Systems 96 (2016) 29-39.

[35] Z.S. Xu, J. Chen, Some models for deriving the priority weights from interval fuzzy preference relations. European Journal of Operational Research 184(1) (2008) 266-280.

[36] Y.J. Xu, F. Herrera, H.M. Wang, A distance-based framework to deal with ordinal and additive inconsistencies for fuzzy reciprocal preference relations. Information Sciences 328 (2016) 189-205.

[37] G.Q. Zhang, Y.C. Dong, Y.F. Xu, Linear optimization modeling of consistency issues in fuzzy group decision making. Expert Systems with Applications 39 (2012) 2415-2420.

[38] Y. Zhang, H.X. Ma, Q. Li, B.H. Liu, J. Liu, Conditions of two methods for estimating missing preference information. Information Sciences 279 (2014) 186-198. 


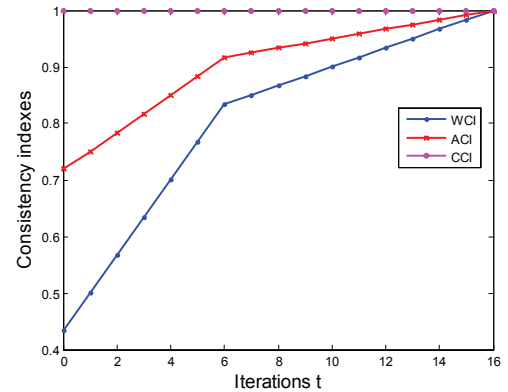

(a) $\alpha=0.05$

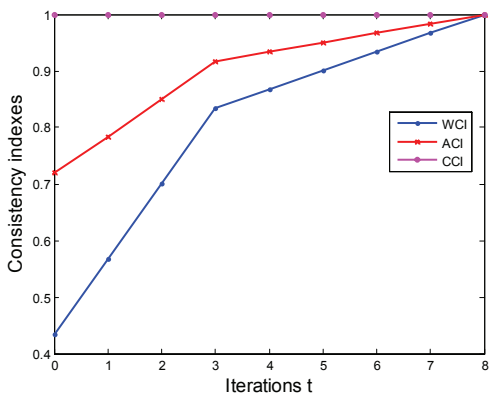

(b) $\alpha=0.1$

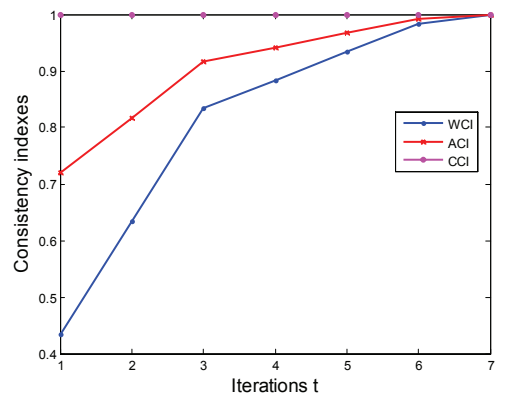

(c) $\alpha=0.15$

Figure 1. The process to improve the consistency indexes of $\widetilde{V}_{1}$ in Algorithm 2

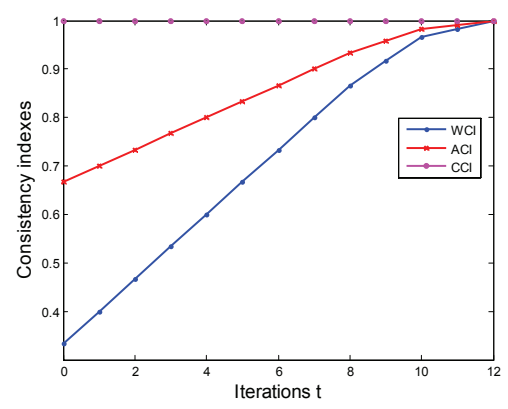

(a) $\alpha=0.05$

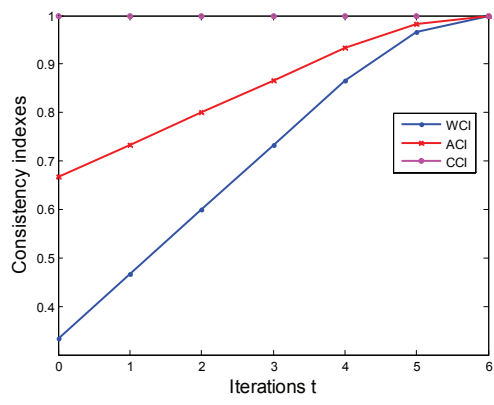

(b) $\alpha=0.1$

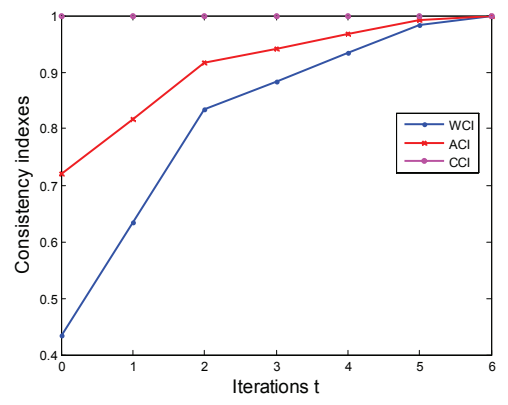

(c) $\alpha=0.15$

Figure 2. The process to improve the consistency indexes of $\widetilde{V}_{2}$ in Algorithm 2

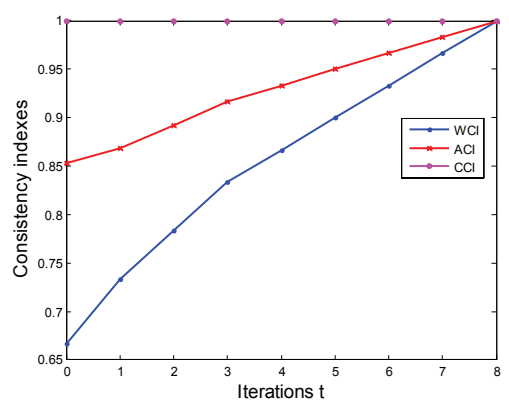

(a) $\alpha=0.05$

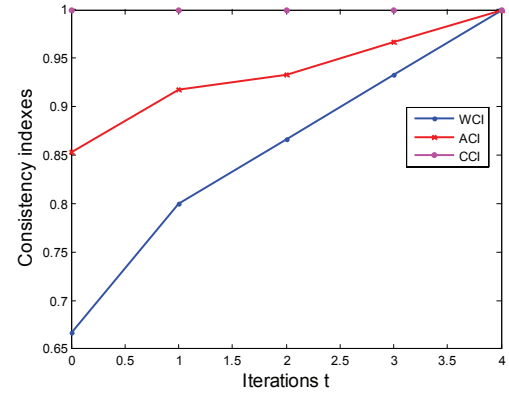

(b) $\alpha=0.1$

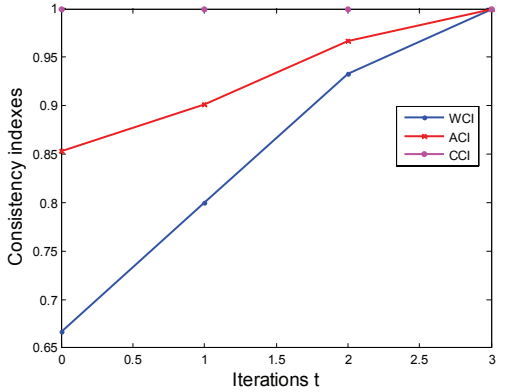

(c) $\alpha=0.15$

Figure 3. The process to improve the consistency indexes of $\widetilde{V}_{5}$ in Algorithm 2

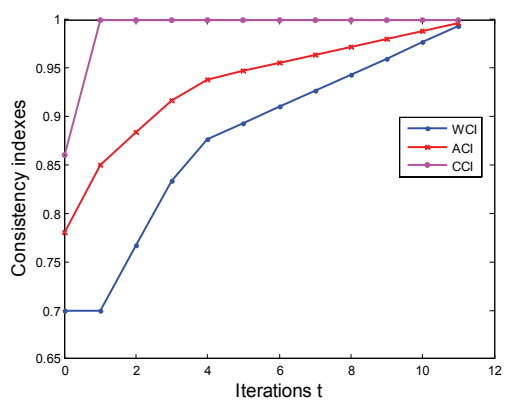

(a) $\alpha=0.05$

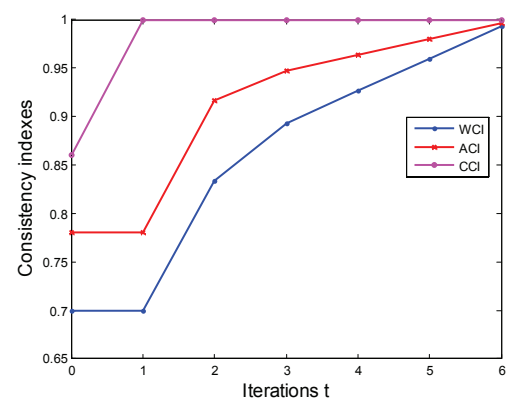

(b) $\alpha=0.1$

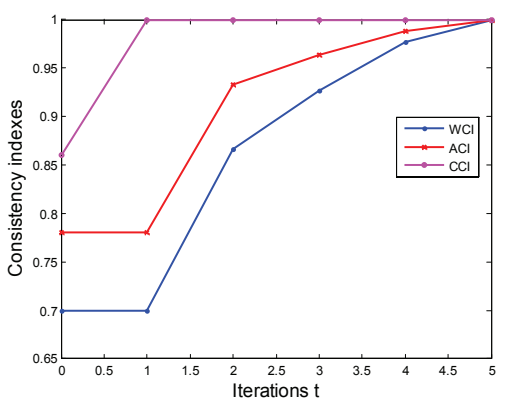

(c) $\alpha=0.15$

Figure 4. The process to improve the consistency indexes of $\widetilde{V}_{6}$ in Algorithm 2 